\title{
Editorial
}

\section{Impact of Nosocomial Infections on Outcome: Myths and Evidence}

\author{
Jordi Rello, MD, $\mathrm{PhD}$
}

The flight of Icarus from Crete to escape the tyranny of Minos, and his fall to the sea when he flew too close to the sun, is one of the best known of the Greek myths. At the end of the 20th century, it is an undisputed fact that patients with nosocomial infections (NI) have high crude mortality and that most patients who die in the intensivecare unit (ICU) die of infection. Multiple clinical trials pursuing survival beyond 28 days after the life-threatening infectious event repeatedly have ended in failure. The drawbacks of crude mortality are its failure to adjust for severity and its failure to identify related deaths, but the infectious event is likely to be a major contributor to death. Nevertheless, the exact contribution of the initiating event (or its severity) and the amount of excess mortality that can be attributed to the development of NI are issues that are understood only partially.

Recent data from prospective studies ${ }^{1}$ of surveillance of $\mathrm{NI}$ in the ICU setting show that $35.1 \%$ of all ICU-acquired bacteremias occurred in patients with intravascular catheters, and it is believed that most primary episodes are secondary to these devices. ${ }^{2}$ Catheter-related (CR) bloodstream infections (BSIs) can be associated with a very wide spectrum of severity, ranging from fever that disappears after catheter withdrawal to endocarditis or septic thrombophlebitis, requiring surgical therapy and prolonging length of stay (LOS). Pittet et al, ${ }^{3}$ in a surgical ICU, reported that the estimated mortality rate for nosocomial BSIs was $35 \%$ (95\% confidence interval $\left[\mathrm{CI}_{95}\right], 25 \%-45 \%$ ). Although intravenous catheters were responsible for less than $25 \%$ of BSIs, ${ }^{4}$ these findings have been generalized, and the myth that all NI, including CR BSI, present high mortality is still widely believed.

The article by Soufir et $\mathrm{al}^{5}$ in this issue of the Journal is a provocative and well-designed case-control study that evaluates whether these infections are responsible for excess mortality in critically ill patients. Major complications were observed in more than one half of exposed patients, and their attending physicians reported that $21 \%$ of these patients died as a consequence of the CR BSI episode. Notably, though, after appropriate matching, no association between the development of CR BSI and increased ICU mortality could be detected. It could be argued that their findings may be biased by the exclusion of most episodes caused by coagulase-negative staphylococci (CNS), but this limitation in fact would contribute to overemphasizing the mortality in the current study population and thus add strength to their conclusion.

In our experience, in an ongoing study involving up to 2,000 patients with over 48 hours of stay in a medicalsurgical ICU, $63.2 \%$ of episodes of CR BSI were caused by CNS. Fifty-seven new episodes of CR BSI were documented, representing an incidence of 2.85 episodes per $100 \mathrm{ICU}$ admissions (data not shown). Forty-nine cases were matched to uninfected controls based upon a number of factors, including the Acute Physiology, Age, and Chronic Health Evaluation (APACHE) II score at admission, underlying disease, age, and prior days of exposure to risk. Six of 31 patients (19.3\%) with CNS bacteremia died in the hospital, whereas crude hospital mortality was $27.8 \%(5 / 18)$ in the group of pathogens other than CNS. As in Soufir et al's study, no significant differences in mortality were found between exposed and unexposed patients.

The first implication of this (ie, that CR BSI may not have an attributable mortality) is important in that it calls into question the myth that as many as 50,000 people die of BSI yearly in the United States. ${ }^{6}$ The current evidence con-

This study was supported in part with a grant from CIRIT (SGR 97/0443). The author thanks Dr. Esteban Reynaga and Ana Ochagavia for their help providing additional information.

99-ED-027. Rello J. Impact of nosocomial infections on outcome: myths and evidence. Infect Control Hosp Epidemiol 1999;20:392-394. 
tradicts earlier suggestions that BSI is associated with high attributable mortality and that some of these deaths conceivably could be avoided by implementing specific preventative measures on patients with intravascular lines. In my opinion, the attributable mortality found in prior studies of NI may have been overvalued by deficiencies in study design, including inadequate adjustment for confounding factors. In contrast, the lower figures reported in recent studies rest on more appropriate assessment of severity and matching.

In 1986 , Forgacs et $\mathrm{al}^{7}$ evaluated bacteremia in the ICU over a 15-year period and found that mortality was $60.4 \%$ in patients with BSI, compared with only $13.1 \%$ in those without BSI. In 1991, Smith et $\mathrm{al}^{8}$ were the first to attempt to control for severity of illness by matching patients based upon predicted mortality estimated by the APACHE II scoring system. The bacteremic group had a crude mortality of $82.4 \%$, whereas the control group had an observed mortality of $52.9 \%$, yielding an attributable mortality of $29.5 \%$. This figure was similar to the attributable mortality (35\%) estimated by Pittet et $\mathrm{al}^{3}$ in 1994 . My main criticism of this study regards matching: exposure to risk was not always equivalent, because some matched controls had shorter LOS than their respective cases when developing BSI. Clearly, a potential case who developed BSI after 20 days of stay cannot be matched with a potential control who was discharged from the ICU before this period of time, because the effect will be to overestimate mortality and LOS for cases.

An outstanding aspect of the study by Soufir et $\mathrm{al}^{5}$ is that it is the first to match patients with CR BSI according to the severity of illness at the onset of bacteremia rather than on admission, as other authors have done. ${ }^{3,7,8}$ This makes sense, because the mean ( \pm standard deviation) duration from ICU admission to onset of CR BSI was $16.8 \pm 9.6$ days, and the severity of illness could have changed substantially prior to infection. Similarly, it is well known in the analysis of survival in patients with sepsis that the predicted mortality based on scores determined at ICU admission undervalue the observed mortality. ${ }^{9}$ Indeed, the Mortality Probability Models II prediction based on determining severity 72 hours after admission adjusted better for observed mortality. These observations recently have been confirmed by our group, demonstrating that the degree of severity of illness at diagnosis of pneumonia in intubated patients (ventilator-associated pneumonia) is the most important predictor of survival in this population. ${ }^{10,11}$

The current evidence showing that development of CR BSI may represent a marker of severity rather than an independent risk factor for mortality also confirms prior findings suggested by multivariate analysis. In a large prospective study ${ }^{12}$ involving 1,745 patients with nosocomial BSI at a single tertiary-care hospital, isolation of Candida species was the only independent microbiological predictor of mortality. Indeed, among primary BSIs, CR infections were associated with a significantly lower risk (odds ratio, $0.58 ; \mathrm{CI}_{95}, 0.39-0.86$ ) of death in the study population. In addition, BSI due to CNS (the most common etiology asso- ciated with CR BSI) tended to predict a favorable outcome and therefore relatively low mortality. Similarly, in a recent multicenter study on BSI acquired in the ICU involving 30 hospitals in Spain, Vallés et $\mathrm{al}^{13}$ reported that, in comparison with other sources, particularly intra-abdominal infection or pneumonia, intravascular catheters were the source associated with the lowest impact on survival. Consequently, the possibility of reducing the risk of death by preventing CR BSI in the ICU seems unrealistic.

However, these infections would have great public health importance in an era of cost containment if they were associated with an increased LOS and the expenditure of more resources. CR BSI acquired in the ICU significantly affected the in-hospital outcome in survivors in our study population, even after controlling for severity of illness. The excess LOS was $19.6 \pm 49.2\left(\mathrm{CI}_{95},-1.1-40.4\right)$ days in survivors, representing an additional cost of $\$ 3,470$ per CR BSI survivor in the ICU. Interestingly, this effect was particularly strong among surviving patients from episodes caused by CNS, with an excess length of hospital stay of 31 days $(P=.02)$. Pittet et al, in their study of BSI, ${ }^{4}$ reported that the infection also was associated with doubled LOS in the ICU, an excess LOS of 24 days in survivors, a significant economic burden. In the light of these figures, I believe that the annual cost arising from the infectious complications of CR BSI in the ICU justifies new efforts to reduce the incidence of these complications. ${ }^{14,15}$

The debate about the impact of NI on outcome will continue, but current evidence is providing a new perspective on the myth that its effect is decisive. The study by Soufir et al, as well as other studies in critically ill patients, gives clear-cut evidence of the key role of severity of illness on evaluating outcome in patients with NI. While we know that assessment of severity done on admission can predict the overall survival of a cohort of patients, we have learned now that this analysis no longer should be used to measure the probability of surviving an episode of NI developed some days later. We also should refine the methods of matching to improve our understanding of the contribution of NI on outcome: unexposed patients should be matched on the day their individual pair developed the infection by the degree of severity of illness documented immediately before the onset of the infectious complication. Traditional approaches that simplify the process of matching no longer should be considered appropriate.

\section{REFERENCES}

1. Rello J, Ricart M, Mirelis B, Quintana E, Gurgui M, Net A, et al. Nosocomial bacteremia in a medical-surgical intensive care unit: epidemiologic characteristics and factors influencing mortality in 111 episodes. Intensive Care Med 1994;20:94-98.

2. Rello J, Quintana E, Mirelis B, Gurgui M, Net A, Prats G. Polymicrobial bacteremia in critically ill patients. Intensive Care Med 1993;19:22-25.

3. Pittet D, Tarara D, Wenzel RP. Nosocomial bloodstream infection in critically ill patients: excess length of stay, extra costs, and attributable mortality. JAMA 1994;271:1598-1601.

4. Pittet D, Wenzel RP. Nosocomial bloodstream infection in the critically ill. JAMA 1994;272:1820.

5. Soufir L, Timsit J-F, Mahe C, Carlet J, Regnier B, Chevret S. Attributable morbidity and mortality of catheter-related septicemia in critically ill patients: a matched, risk-adjusted cohort study. Infect Control Hosp Epidemiol 1999;20:396-401. 
6. Wenzel RP, Edmond MB. The evolving technology of venous access. $N$ Engl J Med 1999;340:48-50.

7. Forgacs IC, Eykyn SJ, Bradley RD. Serious infection in the intensive therapy unit: a 15-year study of bacteremia. QJM 1986;60:773779 .

8. Smith RS, Meixler S, Simberkoff M. Excess mortality in critically ill patients with nosocomial bloodstream infection. Chest 1991;100: 164-167.

9. Le Gall JR, Lemeshow S, Leleu G, Klar J, Huillard J, Rué M, et al. Customized probability models for early severe sepsis in adult intensive care patients. JAMA 1995;273:644-650.

10. Rello J, Rué M, Jubert $P$, Muses G, Sońora $R$, Vallés J, et al. Survival in patients with nosocomial pneumonia: impact of the severity of illness and the etiologic agent. Crit Care Med 1997;25:1862-1867.
11. Rello J, Vallés J. Mortality as an outcome in hospital-acquired pneumonia. Infect Control Hosp Epidemiol 1998;19:795-797.

12. Pittet D, Li N, Woolson RF, Wenzel RP. Microbiological factors influencing the outcome of nosocomial bloodstream infections: a 6 -year validated, population-based model. Clin Infect Dis 1997;24:1068-1078.

13. Vallés J, León C, Alvarez-Lerma F. Nosocomial bacteremia in critically ill patients: a multicenter study evaluating epidemiology and prognosis. Clin Infect Dis 1997;24:387-395.

14. Segura M, Alvarez-Lerma F, Tellado JM, Jimenez-Ferreres J, Oms L, Rello J, et al. A clinical trial on the prevention of catheter-related sepsis using a new hub model. Ann Surg 1996;223:363-369.

15. Darouiche RO, Raad II, Heard SO, Thornby JI, Wenker OC, Gabrielli A et al. A comparison of two antimicrobial-impregnated central venous catheters. N Engl J Med 1999;340:1-8. 